\title{
The Rap1 Guanine Nucleotide Exchange Factor C3G Is Required for Preservation of Larval Muscle Integrity in Drosophila melanogaster
}

\author{
Margret Shirinian ${ }^{1}$, Caroline Grabbe', Milica Popovic ${ }^{2}$, Gaurav Varshney ${ }^{1}$, Fredrik Hugosson ${ }^{1}$, Hans \\ Bos $^{2}$, Holger Rehmann ${ }^{2}$, Ruth H. Palmer ${ }^{1 *}$
}

1 Department of Molecular Biology, Umeå University, Umeå, Sweden, 2 Department of Physiological Chemistry, Centre for Biomedical Genetics and Cancer Genomics Centre, University Medical Center, Utrecht, The Netherlands

\begin{abstract}
C3G is a guanine nucleotide exchange factor (GEF) and modulator of small G-protein activity, which primarily acts on members of the Rap GTPase subfamily. Via promotion of the active GTP bound conformation of target GTPases, C3G has been implicated in the regulation of multiple cellular and developmental events including proliferation, differentiation and apoptosis. The Drosophila C3G orthologue exhibits a domain organization similar to that of vertebrate C3G. Through deletion of the C3G locus, we have observed that loss of C3G causes semi-lethality, and that escaping adult flies are characterized by a reduction in lifespan and general fitness. In situ hybridization reveals C3G expression in the developing embryonic somatic and visceral muscles, and indeed analysis of C3G mutants suggests essential functions of C3G for normal body wall muscle development during larval stages. C3G mutants display abnormal muscle morphology and attachment, as well as failure to properly localize $\beta$ PS integrins to muscle attachment sites. Moreover, we show that C3G stimulates guanine nucleotide exchange on Drosophila Rap GTPases in vitro. Taken together, we conclude that Drosophila C3G is a Rap1-specific GEF with important functions in maintaining muscle integrity during larval stages.
\end{abstract}

Citation: Shirinian M, Grabbe C, Popovic M, Varshney G, Hugosson F, et al. (2010) The Rap1 Guanine Nucleotide Exchange Factor C3G Is Required for Preservation of Larval Muscle Integrity in Drosophila melanogaster. PLoS ONE 5(3): e9403. doi:10.1371/journal.pone.0009403

Editor: Christos Samakovlis, Stockholm University, Sweden

Received September 22, 2009; Accepted February 4, 2010; Published March 3, 2010

Copyright: (c) 2010 Shirinian et al. This is an open-access article distributed under the terms of the Creative Commons Attribution License, which permits unrestricted use, distribution, and reproduction in any medium, provided the original author and source are credited.

Funding: MP was supported by TI Pharma Project T3-106 and HR by the Chemical Sciences of the Netherlands Organization for Scientific Research (NWO-CW) RHP is a Swedish Cancer Foundation Research Fellow and was supported by the Swedish Research Council (621-2003-3399), the Swedish Cancer Research Society (09 0741) and the Association for International Cancer Research. The funders had no role in study design, data collection and analysis, decision to publish, or preparation of the manuscript.

Competing Interests: The authors have declared that no competing interests exist.

* E-mail: Ruth.Palmer@ucmp.umu.se

\section{Introduction}

The ability of a cell to accurately respond to external signals in different developmental contexts relies on the integration of multiple sets of signaling pathways, which in many cases are highly conserved through evolution. A key event of such cellular signaling is the ligand-mediated activation of receptor tyrosine kinase (RTK) family proteins, leading to the recruitment of a multitude of proteins that function to transmit signals to the proper downstream targets. Essential players in this process include protein and lipid kinases, adaptor and scaffolding molecules, as well as members of the small GTPase superfamily. Small GTPases are monomeric GTP-binding proteins of $20-25 \mathrm{kDa}$, which act as molecular switches during diverse cellular and developmental events, including proliferation, differentiation, apoptosis and control of the cytoskeleton [1]. The rather large superfamily of small GTPases is subdivided, based on structure and function, into the Rho, Rab, Ran, Arf/Sar and Ras (which is further divided into Ras/Ral/Rap subfamilies) GTPase families. GTPases cycle between an inactive GDP-bound and an active GTP-bound conformation, a process which is regulated by the concerted action of activating guanine nucleotide exchange factors (GEFs) and inhibiting GTPase activating proteins (GAPs) [2]. In analogy to the small GTPases, the human genome encloses a large number of selective GEF families, in which unique combinations of the GEF domain with specific protein modular domains provide activity, which is specific towards the corresponding GTPase (Bos, 2007). Similar rules also apply to vertebrate GAP proteins.

The Rap family of GTPases, and thus their associated regulators, are primarily involved in regulating cell-cell junction formation, cell adhesion to extracellular matrix and polarity [1]. One of the GEFs that has been assigned specifically to Rapl is $\mathrm{C} 3 \mathrm{G}$, a multidomain protein which was originally isolated as a binding partner of the v-GRK adaptor molecule [3], interacting with the CRK SH3 domain via four proline-rich regions in the central region of the molecule [3,4]. Later on, the C3G CDC25Ras exchange motif (CDC25-REM) was reported to stimulate guanine nucleotide exchange on at least two Ras family members, Rapl and R-Ras in vitro $[5,6]$.

In vivo studies in mouse models have shown that $\mathrm{C} 3 \mathrm{G}$ is essential during early embryogenesis and that C3G null mutant embryos die around day 5.0 of gestation, thereby creating difficulties in the study of C3G function during development [7]. A hypomorphic allele, $C 3 G^{s t}$, which produces less than $5 \%$ of normal C3G protein levels, allows the survival of $C 3 G^{\text {gt/gt }}$ mutants to embryonic day 14 (E14.5) [8]. C3 $G^{\text {gt/gt }}$ mutant mice die of hemorrhaging due to 
vascular defects, suggesting a role for C3G in vascular myogenesis, in keeping with the lack of correctly developed supporting cells in $C 3 G^{\text {tt } / g t}$ animals. Moreover, C3G has recently been implicated in the regulation of the size of the cerebral cortex neural precursor population, and mice lacking $\mathrm{C} 3 \mathrm{G}$ exhibit excessive proliferation in the cortical neuroepithelium [9]. Although C3G has several proposed functions in vertebrates, its physiological and biological role in Drosophila is unknown [10,11,12]. Previously, Drosophila C3G has been shown to have a genetic interaction with Rapl and with components of the Ras-MAPK pathway [13], however direct evidence of Drosophila C3G GEF activity and specificity is lacking. In addition, the absence of $C 3 G$ mutants has precluded an evaluation of the importance of $\mathrm{C} 3 \mathrm{G}$ protein function in the fly.

In order to increase the understanding of $\mathrm{C} 3 \mathrm{G}$ in vivo, we have generated Drosophila C3G null mutants and concluded that loss of C3G results in semi-lethality, with escaping adults characterized by a shorter life span and reduced general fitness. While in situ hybridization depicts C3G expression in embryonic CNS, somatic and visceral muscles, our analysis of Drosophila C3G mutant embryos has failed to reveal any major embryonic developmental defects in any of these tissues. However, when investigating the larval development of Drosophila C3G mutants, we detected substantial disorganization of the larval muscle architecture, as well as defects in integrin localization at muscle attachment sites. In support of a role of $\mathrm{C} 3 \mathrm{G}$ in somatic muscles, we have also observed that misexpression of an activated form of $\mathrm{C} 3 \mathrm{G}$ results in defective muscle structure and muscle-muscle detachment. To study the function of $\mathrm{C} 3 \mathrm{G}$ in detail at the molecular level, we have performed guanine nucleotide exchange assays, providing conclusive evidence that Drosophila C3G acts as a GEF for Drosophila Rapl in vitro. Moreover, Drosophila C3G also exhibits GEF activity towards human Rapl but not H-Ras. In conclusion, we propose that C3G is an accessory component of the Drosophila musculature, essential for the proper localization of integrins at muscle-muscle and muscle-epidermis attachment sites and important for maintaining muscle integrity during larval stages.

\section{Methods}

\section{Fly Stocks}

Standard Drosophila husbandry procedures were applied. Drosophila strains were maintained on standard potato-meal medium, and raised and crossed at room temperature unless otherwise stated. The PiggyBac elements used in the study were from the Harvard Exelixis stock collection [14]. The various $U A S$ $C 3 G$ transgenic flies $\left(U A S-C 3 G^{W T}, U A S-C 3 G^{C A}\right.$ and $\left.U A S-C 3 G^{D \mathcal{N}}\right)$ were kindly provided by Dr U. Gaul [13]. The Zasp-GFP reporter was obtained from Bloomington (stock 6338).

\section{Generation of Drosophila C3G Deletion Mutants}

The Drosophila $C 3 G$ deletion mutant $\left(\triangle C 3 G^{M S}\right)$ was generated by heat shock FLP-induced FRT-mediated recombination of the transposable elements RBe03301 and XPd00064 [14]. $\Delta C 3 G^{M S}$ deletion mutants were identified and verified by genomic PCR using the primers, AACAAATTGTTGTTATGCGT for the 5' side and AGAATGGGGTGTGCCGTAAG for the $3^{\prime}$ side. Drosophila Miple2 was used as a control for the genomic PCR using the primers CGAAATAAAACACTCTACG for the $5^{\prime}$ side and CAAATCGCCATTGGAAACTC for the $3^{\prime}$ side. Mutants were additionally confirmed by Southern Blot analysis.

\section{Drosophila DNA Preparation and Southern Blotting}

Genomic DNA was prepared using standard techniques. Genomic DNA, digested with HindIII, EcoRI (New England
Biolabs) was electrophoresed on $1 \%$ agarose gel and blotted onto Hybond N+ filter (GE Healthcare). The filter was subsequently analyzed using Digoxigenin-labeled (DIG) Drosophila C3G specific DNA probes, which were detected by the DIG detection chemiluminescent assay (Roche). Primers used for making the probe were CTCGATCGACGCGCGGCACGTGTT for the 5' side and ATTGGAACTGCAGTCGAGGTCGGA for the $3^{\prime}$ side.

\section{Drosophila RNA Preparation and Reverse Transcription- PCR Analysis}

RNA was isolated from adult Drosophila using RNA isolation kit (Bio-Rad). Reverse transcription was performed using the Superscript II reverse transcriptase (Invitrogen) and primers for shifted mRNA. The primers used were GGGGGATTTTTCAAGTGTCGAAAC for the $5^{\prime}$ side and CGTTTTGTCGCTGTTGCTGTTGTT for the $3^{\prime}$ side.

\section{In situ Hybridisation}

A DIG RNA labeling kit (Roche) was applied to generate DIGlabeled RNA probes, using full-length Drosophila C3G cDNA (RE10624, accession number BT010019) as template. In situ hybridization of whole-mount wild type Drosophila embryos was carried out as described [15].

\section{Antibody Production against Drosophila C3G}

For the preparation of Drosophila C3G antiserum, DNA encoding C3G amino acids 1- 155 was generated by PCR and subcloned into pETM-11. The reading frame of the His-C3G sequence was subsequently confirmed by sequence analysis. Recombinant His-C3G fusion protein was purified form Echerichia coli (BL21 (DE3)) bacterial lysates by standard protocols using NiNTA agarose (Qiagen) and subsequently used for immunization of guinea pigs.

\section{Drosophila Protein Isolation and Western Blotting}

Adult Drosophila flies of the indicated genotypes were ground in Lysis Buffer consisting of $50 \mathrm{mM}$ HEPES pH 7.4, $150 \mathrm{mM} \mathrm{NaCl}$, 1 mM EDTA, 1 mM EGTA, 1\% Triton-X-100, 10\% Glycerol, $25 \mathrm{mM} \mathrm{NAF}, 10 \mu \mathrm{M} \mathrm{ZnCl} 2$ and a protease inhibitor cocktail (Complete, Roche). Lysates were cleared by centrifugation and protein concentrations determined using the Bio-Rad protein assay. Protein samples were separated on SDS-PAGE and transferred to a polyvinylidene difluoride membrane (Millipore). Membranes were blocked in 5\% BSA for 1 hour, prior to incubation with either guinea pig anti-C3G, or mouse anti- $\alpha$ Tubulin (Sigma) antibodies and detection by ECL (GE Healthcare).

\section{Lethality and Life Span Determination}

For life span experiments, flies were collected during 24 hours and aged at the temperatures indicated. Flies were transferred daily onto fresh food and counted. The lethality determination was performed by placing 100 embryos of the indicated genotypes onto apple juice plates. Embryos developing into larvae and larvae enclosing into adult flies were counted.

\section{Immunostaining and Antibodies}

Embryos were fixed and immunostained as previously described [16]. Larval muscles were heated at $60^{\circ} \mathrm{C}$ for 10 seconds, fixed in $4 \%$ formaldehyde, blocked in $5 \%$ NGS overnight at $4{ }^{\circ} \mathrm{C}$ and stained according to standard procedures. The following primary antibodies were used: rabbit anti-dAlk (1:1000) [17], guinea pig anti-Alk (1:1000) [18], rabbit anti- $\beta 3$-tubulin (1:3000) [19], mouse 
anti-myc (1:1000, 9E10), rabbit anti-myc (1:250, Sigma), mouse anti- $\alpha$ GFP $(1: 1000$, Clontech), mouse anti- $\beta$ PS integrin (CF.6G11, 1:20, Developmental Studies Hybridoma Bank), Rhodamine Phalloidin, Phalloidin Alexa flour 488, Phalloidin Alexa flour 647 (Molecular Probes). The following secondary antibodies were used; biotin-conjugated anti-rabbit IgG (1:500, Vector Laboratories), Cy3 goat anti-mouse (1:1000, Jackson), Cy2 goat anti-mouse (1:1000, GE Healthcare), Cy2 goat anti-rabbit (GE Healthcare), Cy3 goat anti-rabbit (GE Healthcare), Cy2 donkey anti-rat (Jackson ImmunoResearch). Embryos were cleared in methyl salicylate (Sigma) before visualization. Embryo staging was carried out according to Campos-Ortega and Hartenstein [20]. Larval muscles were mounted in Vectashield (Vector Laboratories).

\section{TUNEL Assay}

TUNEL assay was performed according to the manufacturer's protocol (In Situ Cell Death Detection Kit, Fluorescein, Roche). Larval muscles were dissected and fixed in $4 \%$ paraformaldehyde, before being washed and permeabilized in PBS-T $(0.1 \%$ Triton X100 in PBS). Muscles were subsequently stained with the TUNEL reaction mixture for 1 hour at $37^{\circ} \mathrm{C}$ in the dark. Samples were rinsed three times with $\mathrm{PBS}$ and analyzed directly using a fluorescence microscope. As positive control permeabilized cells were incubated with DNase $\mathrm{I}\left(3 \mathrm{U} \mathrm{ml}^{-1}\right.$ in $50 \mathrm{mM}$ Tris- $\mathrm{HCl}$, $\mathrm{pH} 7.5,1 \mathrm{~g} \mathrm{l}^{-1} \mathrm{BSA}$ ) for 10 minutes at room temperature to induce DNA strand breaks. As negative control permeabilized cells were incubated in TUNEL reaction mixture without addition of the terminal transferase.

\section{Recombinant Proteins for the Guanine Nucleotide Exchange Assay}

Full-length Drosophila Rap1 and Rap2L cDNA clones (RE42418 and RE63021, respectively) were obtained from DGRG (Drosophila Genomics Resource Center). Drosophila C3G RNA was isolated (Bio-Rad) and cloned by RT-PCR (Invitrogen). Fragments were cloned using BamHI/EcoRI for (C3GGEF) and EcoRI/NotI for (Drosophila Rapl and Rap2L). The following constructs were generated; pGEX4T3:Rap1 (aa 1-167), pGEX4T3-1:Rap2L (aа 1-167), pGEX4T-1:C3GGEF (aa 1332-1565). Rap1B (Homo sapiens, aa 1-167) and H-Ras (Homo sapiens, aa 1-166) were expressed from ptac; Rap2B (Homo sapiens, aa 1-166) was expressed from pGEX4T3, C3G (Homo sapiens, aa 830-1078) expressed from pET15 and Sos (Homo sapiens, aa 564-1049) from pET28.

\section{Guanine Nucleotide Exchange Assay}

To test the GEF activity of Drosophila C3G, human C3G and Sos on Drosophila Rap1 and Rap2, human Rap1B, human Rap2B and human H-Ras purified GTPases were preloaded with fluorescent mant-GDP (2'-/3'-O-(N'-Methylanthraniloyl)guanosine-5'-O-diphosphate) by incubating the GTPase in the presence of EDTA and an excess of mant GDP. The EDTA and free nucleotides were removed by gelfiltration [21]. Due to the high intrinsic off rate of Drosophila Rap2, Drosophila Rap2 was loaded by incubating 200nM mant-GDP with $200 \mathrm{nM}$ Drosophila Rap2 until a stable fluorescent signal was obtained. The reaction was started by adding an excess of GDP and GEF and the fluorescence signal was measured over time (excitation wavelength is $366 \mathrm{~nm}$ and emission $450 \mathrm{~nm})$. The buffers used are (50mM TrisHCL, pH 7.5, $500 \mathrm{mM} \mathrm{NaCl}$ (except for the Ras/Sos reaction, where buffers contained $100 \mathrm{mM} \mathrm{NaCl}$ ), $5 \mathrm{mM} \mathrm{MgCl}_{2}, 5 \mathrm{mM}$ DTT, $5 \%$ glycerol). The reaction mixture consisted of 200nM of GTPase, 150nM RapGEF6 or 1500nM Drosophila C3G, human C3G or Sos, and $27 \mu \mathrm{M}$ of GDP.

\section{Confocal Imaging}

Confocal imaging was performed by using a Nikon confocal microscope C1, (Kangawa, Japan) fitted with $\mathrm{Ar}, \mathrm{He} / \mathrm{Ne}$, and blue diode lasers. Digitized confocal images were assigned red and green pseudocolors for Cy3/Alexa Fluor 594 and FITC/Alexa Fluor 488 respectively. $40 \mathrm{X}$ and $60 \mathrm{X}$ objectives were used. Captured images were exported as TIFF format files and further processed using Adobe Photoshop 8.0 for figure mounting and labeling purposes. Larval muscle pictures at high magnification were taken using a Leica confocal microscope (TCS-SPE), images were assigned red (532), green (488) and Cy5 (647). At low magnification, images of larval body wall muscles were captured using an Axia Imager.Z2 system (Zeiss).

\section{Results}

\section{Generation and Characterization of C3G Loss of Function Mutants}

Similar to its mammalian orthologues, the Drosophila C3G protein contains a catalytic CDC25-type GEF domain, in tandem with a conserved GEFN/REM domain, showing $67 \%$ and $39 \%$ identity to its human counterparts, respectively. Strong homology is also displayed within the N-terminal SH3 CRK binding prolinerich regions, suggesting a potential evolutionary conservation of the CRK-G3G interaction (Figure 1A, triangles) [13]. A Drosophila $C 3 G$ loss-of-function mutant $\left(\triangle C 3 G^{M S}\right)$ was generated by FLP/ FRT-mediated recombination of two independent PiggyBac elements flanking the Drosophila C3G locus [14], which resulted in the successful deletion of most of the $C 3 G$ coding region (Figure 1B). $\triangle C 3 G^{M S}$ mutants were initially identified by genomic PCR (Figure 1G) and subsequently verified by Southern Blot (Figure 1D), inverse PCR and sequence analysis (data not shown). Accordingly, analysis of homozygous $\triangle C 3 G^{M S}$ mutant flies by immunoblotting with antibodies raised against $\mathrm{C} 3 \mathrm{G}$ showed a loss of C3G protein in the mutant animals (Figure 1E). To further confirm the specificity of the mutant we investigated the integrity of the neighboring gene on the $3^{\prime}$ side (shifted) by performing RTPCR on mRNA extracted from $\triangle C 3 G^{M S}$ flies. Indeed, shifted mRNA can still be detected in $\triangle C 3 G^{M S}$ mutants (Figure $\mathrm{S} 1$ ). $\triangle C 3 G^{M S}$ mutant flies are viable and fertile, but hatch in significantly reduced numbers, indicating semi-lethality. Analysis of the life span of Drosophila C3G deficient adult flies indicates a considerably shortened life span, with a survival rate of $50 \%$ after 9 days at $29^{\circ} \mathrm{C}$, as compared with 26 days in controls (Figure $2 \mathrm{~A}$ and $\mathrm{B})$. A detailed examination of the viability of $\triangle C 3 G^{M S}$ animals during the course of development revealed that while $70 \%$ of $\triangle C 3 G^{M S}$ mutant embryos developed into larvae, only $32 \%$ eclosed into adult flies (Figure 2C). Taken together, these results suggest that Drosophila $C 3 G$ plays a role in larval fitness and longevity of adult flies, and that $\triangle C 3 G^{M S}$ animals are semi-lethal.

\section{$\triangle C 3 G^{M S}$ Mutant Embryos Do Not Exhibit Any Obvious Embryonic Muscle Defects}

During embryonic stages Drosophila C3G is expressed in the visceral and somatic mesoderm as well as the CNS (Figure 3 and [13]), suggesting a role for C3G during the development of these tissues. In agreement with previous reports [13] we observe that $C 3 G$ is expressed in the founder cells of the developing visceral mesoderm (Figure 3A). Founder cells are essential for the formation of the midgut musculature, and indeed flies lacking these cells - such as mutants for the RTK Alk and its ligand Jeb - do not form a functional midgut $[17,18,22]$. However, investigation of the visceral muscle fusion process in $\triangle C 3 G^{M S}$ mutant animals indicates that $\mathrm{C} 3 \mathrm{G}$ activity is not essential for this process (Figure $\mathrm{S} 2 \mathrm{C}$ and $\mathrm{D}$ ). In 
A.

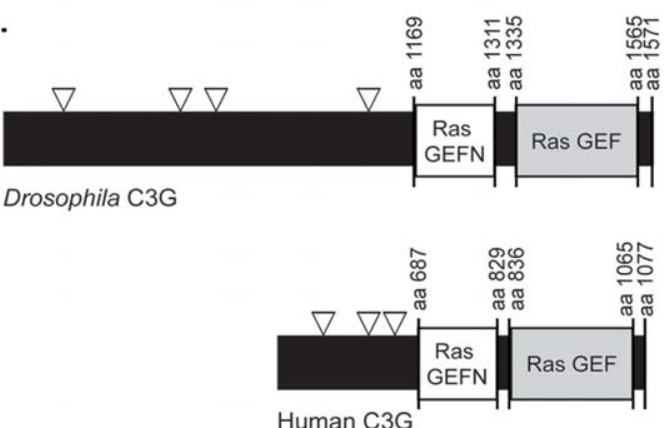

B.

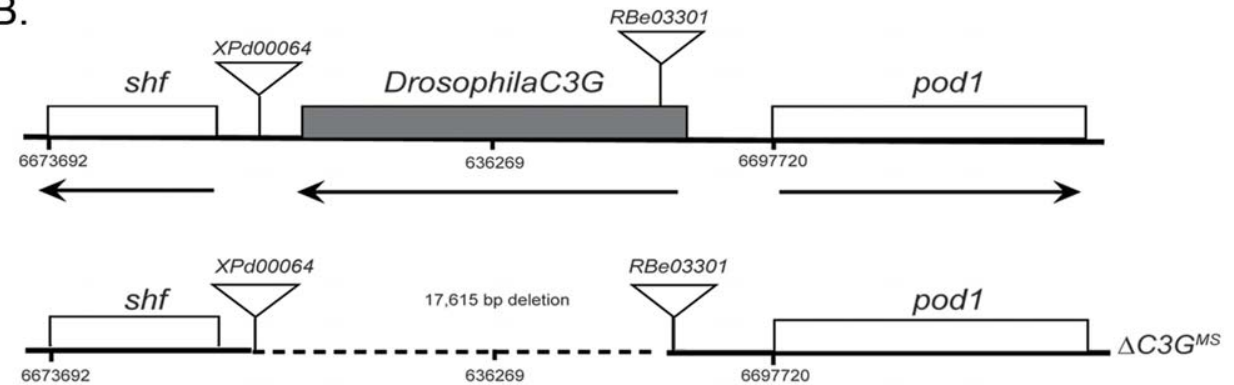

C.

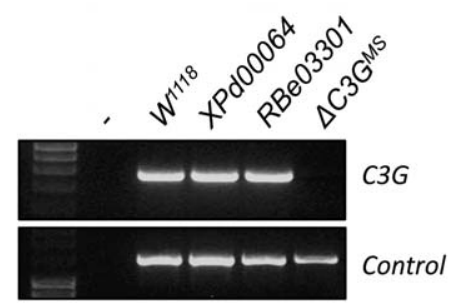

D.

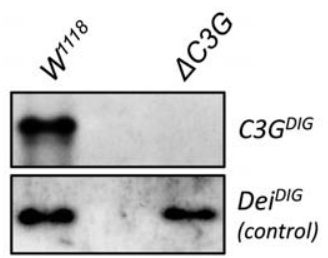

E.

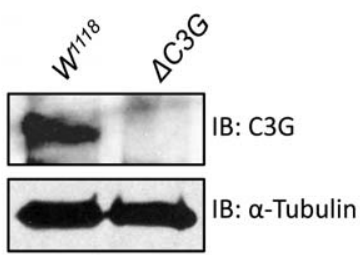

Figure 1. Generation of Drosophila C3G mutant flies. (A) Domain structure of Drosophila and human C3G. C3G consists of a long N-terminal domain (grey box), a catalytic RasGEF domain and a RasGEFN domain, which is believed to have a structural importance rather than catalytic activity; triangles indicate proline rich regions. (B) The Drosophila C3G deletion allele used in this study was generated by FLP/FRT-mediated recombination between two independent transposons (RBe03301 and XPd00064), inserted in the $5^{\prime}$ and the $3^{\prime}$ side of the C3G locus, respectively. This deletion results the deletion of the most of the coding region of the $C 3 G\left(\Delta C 3 G^{M S}\right)$. (C) The $\Delta C 3 G^{M S}$ mutant was verified by genomic PCR (lower panel) and (D) Southern blotting. (E) Western Blot analysis of the indicated genotypes confirms the absence of detectable C3G protein expression in $\Delta C 3 G^{M S}$ mutants.

doi:10.1371/journal.pone.0009403.g001

addition, the analysis of the embryonic somatic musculature of $\triangle C 3 G^{M S}$ mutant embryos, employing anti- $\beta 3$-tubulin antibodies, failed to reveal any obvious defects also in this muscle type (Figure 4A and B). Further, the localization of $\beta$ PS integrins at muscle attachment sites did not display any obvious abnormalities in $\triangle C 3 G^{M S}$ mutant embryos ( Figure $\mathrm{S} 2 \mathrm{~A}$ and $\mathrm{B}$ ). Thus, both the visceral and somatic muscles appear to be grossly normal in $\triangle C 3 G^{M S}$ mutant embryos and we were unable to identify any major defects in embryonic muscle morphology, attachment or migration (Figure 4A, B and Figure S2). Importantly, when analyzing $\Delta C 3 G^{M S}$ mutants at later stages, we could conclude that the morphology of larval and adult guts was also generally normal (data not shown). Taken together, this data indicates that formation of the gut musculature proceeds normally in $\triangle C 3 G^{M S}$ mutant animals, and by extension that Drosophila C3G is not required for gross visceral muscle development.

\section{$\triangle C 3 G^{M S}$ Mutant Larvae Display a Distinct Somatic Muscle Phenotype}

Given the absence of embryonic phenotypes, we continued our studies by examining the larval musculature in $\Delta C 3 G^{M S}$ mutants. To do this, body wall muscles from third instar larvae were stained with phalloidin to visualize the actin cytoskeleton, together with $\beta$ PS integrin, marking muscle attachment sites. A significant proportion $(90 \%, \mathrm{n}=30)$ of $\Delta C 3 G^{M S}$ mutant specimens analyzed showed abnormal muscle architecture of the ventral longitudinal muscles. These experiments were repeated in a double-blind manner with similar results. The $\Delta C 3 G^{M S}$ mutants most 
A.

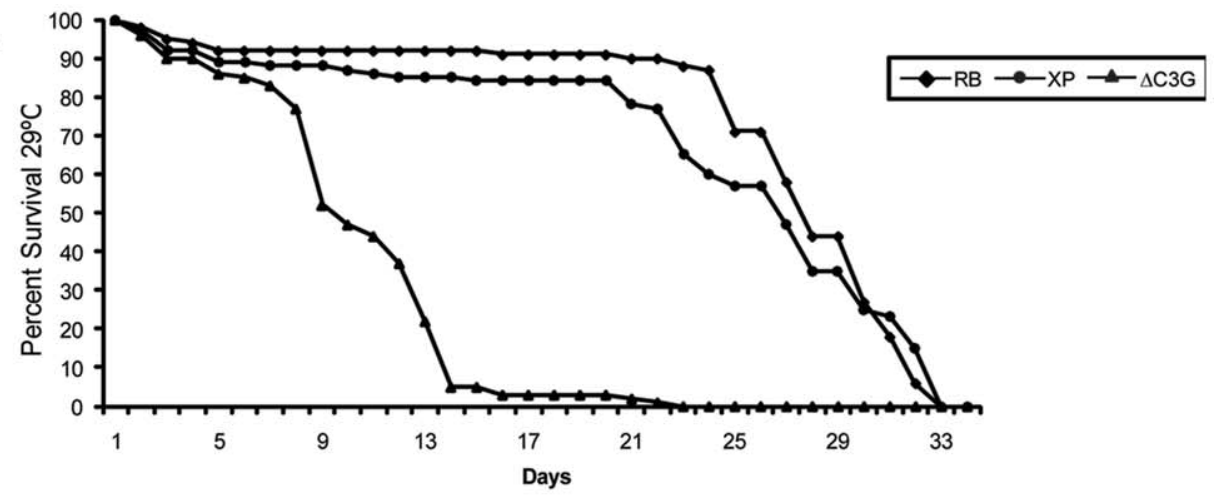

B.

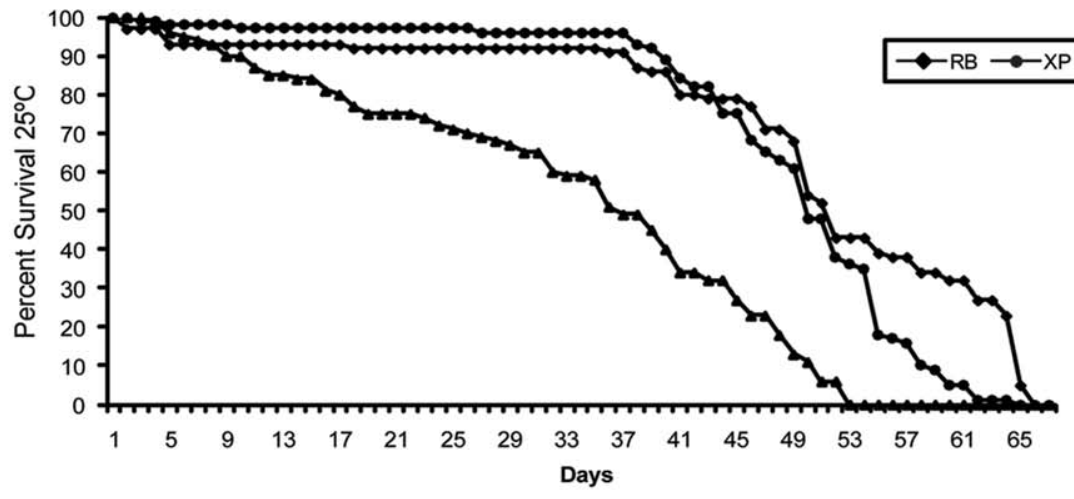

C.

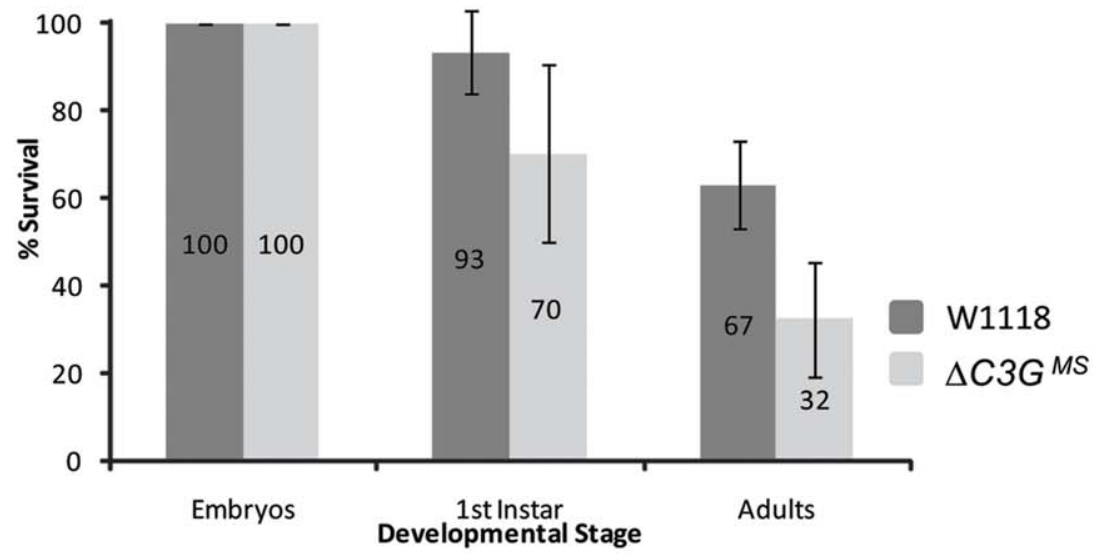

Figure 2. Drosophila C $3 G$ mutants display reduced life span and eclosion rates. (A, B) The life span of $\Delta C 3 G^{M S}$ mutant flies was studied. Flies carrying the starting transposons (RBe03301 (RB) and XPd00064 (XP)) were analyzed in parallel, as controls. $\Delta C 3 G^{M S}$ mutant flies die earlier than control flies, when aged at both $29^{\circ} \mathrm{C}(\mathbf{A})$ and $25^{\circ} \mathrm{C}(\mathbf{B})$. (C) Lethality determination analysis was performed on $\Delta C 3 G^{M S}$ mutant and wild type animals in parallel, starting with 100 embryos of each genotype. Out of these 100 , only $32 \%$ of $\Delta C 3 G^{M S}$ mutants hatched into adults, which is significantly fewer when compared to $67 \%$ of controls.

doi:10.1371/journal.pone.0009403.g002

commonly displayed phenotypes including deformations and detachment of the ventral longitudinal muscles (compare Figure $4 \mathrm{D}$ with wild type in $4 \mathrm{C}$, arrows in $4 \mathrm{D}$ indicate muscle detachment, mistargeting and "muscle thinning"), which was observed in roughly $90 \%$ of the muscle samples analyzed (Figure 4F). While muscle defects were not observed in late embryonic stages, we were able to clearly observe defects in dissected second instar $\Delta C 3 G^{M S}$ mutant larva, suggesting that the phenotype arises after embryogenesis (Figure S3). In addition, a disorganization of the sarcomeric muscle architecture in $\Delta C 3 G^{M S}$ mutant animals can be observed using the Z-band specific reporter Zasp-GFP [23] (Figure 4G and H; arrow). The muscle defects observed were rescued by the reintroduction of wild type $\mathrm{C} 3 \mathrm{G}$ protein expression $\left(U A S-C 3 G^{W T}\right)$ under control of the muscle specific Mef2-Gal4 driver in $\triangle C 3 G^{M S}$ mutants $(75 \%$ rescue; $\mathrm{n}=34$ ) (Figure $4 \mathrm{E}$ and data not shown). 

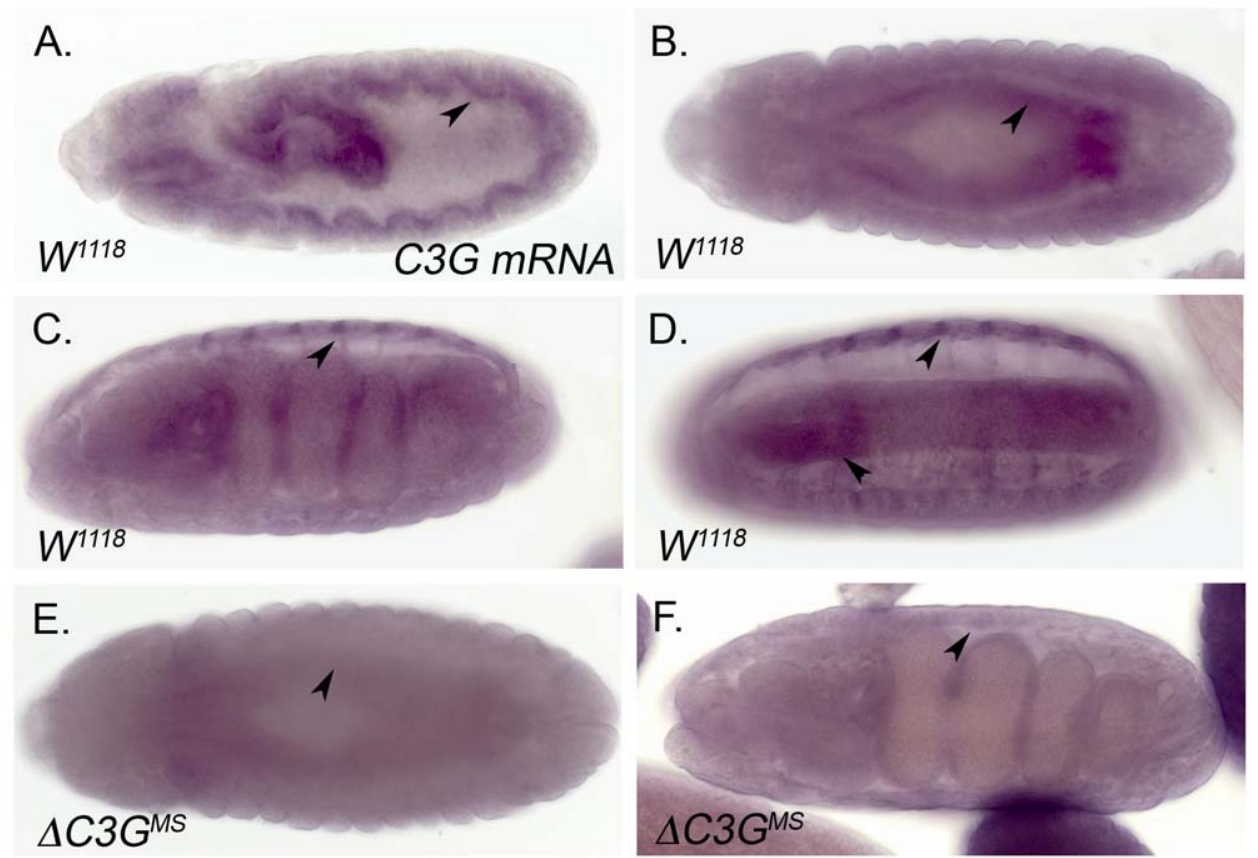

Figure 3. In situ expression pattern of Drosophila C3G. In situ hybridization using a C3G specific RNA probe indicates that C3G mRNA is expressed ubiquitously in Drosophila embryos, with particularly strong expression in the founder cells of the early visceral mesoderm (A), throughout the visceral mesoderm at later stages (B), as well as in somatic muscles (C, D) and the CNS (D). The absence of C3G mRNA could be observed in $\triangle C 3 G^{M S}$ embryos (E, F).

doi:10.1371/journal.pone.0009403.g003

Given the clear defects in the larval musculature of $\Delta C 3 G^{M S}$ animals, we investigated whether any of the molecules known to be important for muscle development in Drosophila was affected in the mutants. Examination of $\beta \mathrm{PS}$ integrin, which is normally localized at muscle attachment sites, revealed an irregular pattern in $\triangle C 3 G^{M S}$ mutants (Figure 5). Whereas $\beta$ PS integrin was indeed found at attachment sites in $\Delta C 3 G^{M S}$ mutants, a significant amount could also be detected throughout the muscle fibers, rather than being concentrated exclusively at the muscle-muscle and muscle-epidermis attachment sites. (Figure $5 \mathrm{C}^{\prime}$ and $\mathrm{D}^{\prime}$ ). These results suggest that although $\mathrm{C} 3 \mathrm{G}$ is not critical for embryonic myogenesis, it appears to be required for the maintenance of muscle morphology and proper attachment during larval stages of muscle development. Notably, this is in agreement with the observation that $68 \%$ of $\triangle C 3 G^{M S}$ mutant larvae fail to hatch into adult flies, which in part may be due to abnormal muscle fiber development.

Morphological defects, similar to those displayed by $\Delta C 3 G^{M S}$ mutant muscles, have also been reported for mutants in genes with established roles in the maintenance of muscle integrity as well as prevention of muscle degeneration and apoptosis, such as the Dystrophin family of proteins [24,25]. To investigate whether Drosophila C3G functions in similar pathways, we wished to examine whether $\triangle C 3 G^{M S}$ mutant larval muscles exhibit increased levels of apoptosis. However, when performing TUNEL assays to detect DNA fragmentation as readout of apoptosis, we were unable to detect any significant difference, when comparing $\Delta C 3 G^{M S}$ to wild type (Figure S4).

\section{Overexpression of Activated C3G Potently Induces Muscle Phenotypes}

In order to better understand the function of C3G during muscle development, we examined the effect of C3G gain-offunction in larval muscles. To do this we utilized the UAS/GAL4 system to specifically express transgenic constructs encoding either wild type, dominant negative (a truncated version of C3G lacking the C-terminal CDC25 catalytic domain) or constitutively activated C3G (a membrane-targeted version of $\mathrm{C} 3 \mathrm{G}$ ) in somatic muscles [13]. The different C3G variants displayed distinct patterns of subcellular localization, as well as effects on muscle morphology. Overexpression of wild type C3G (Mef2-Ga$14>\mathrm{UAS}-\mathrm{C} 3 \mathrm{G}^{\mathrm{WT}}$ ) resulted in an intriguing accumulation of $\mathrm{C} 3 \mathrm{G}$ both in a punctuate manner and on one side of the muscle attachment site (Figure 6A; yellow arrows). The dominant negative C3G (Mef2-Gal4>UAS-C3G ${ }^{\text {DN }}$ ) was localized in a punctuate pattern, spread throughout the muscle fibers with accumulation in perinuclear regions (Figure 6B; yellow arrows). Mef2-Gal4 $>$ UAS$\mathrm{C} 3 \mathrm{G}^{\mathrm{DN}}$ flies were viable and showed no obvious alterations in actin organization (Figure 6B). The strongest effect was undoubtedly observed upon overexpression of activated C3G (Mef2Gal4 $>\mathrm{UAS}^{\mathrm{C}} 3 \mathrm{G}^{\mathrm{CA}}$ ), which when expressed at $25^{\circ} \mathrm{C}$ resulted in embryonic lethality. Examination of somatic muscles of Mef2Gal4>UAS-C3G ${ }^{\mathrm{CA}}$ animals at embryonic stages revealed severe muscle defects (Figure S5), in contrast to embryos expressing wildtype or dominant negative $\mathrm{C} 3 \mathrm{G}$ which were indistinguishable from controls. Expression of UAS-C3 $3 \mathrm{G}^{\mathrm{CA}}$ at $18^{\circ} \mathrm{C}$ allowed development of larvae displaying severe morphological defects and disruption of actin organization in somatic muscle (Figure 6C). Detachment of the ventral longitudinal muscles was also frequently observed (Figure 6C"; arrowhead). Notably, in all cases C3G was found to decorate the muscles in a punctuate pattern, as well as around the muscle nuclei (arrows Figure $6 \mathrm{~B}^{\prime \prime}, \mathrm{C}^{\prime \prime}$ ). Given the mislocalization of $\beta$ PS-integrin in $\triangle C 3 G^{M S}$ mutant muscles we decided to investigate the effect of $\mathrm{C} 3 \mathrm{G}$ overexpression on $\beta \mathrm{PS}-$ integrins. Overexpression of wild type C3G (Mef2-Gal4>UAS$\mathrm{C} 3 \mathrm{G}^{\mathrm{WT}}$ ) resulted in a clear accumulation of $\beta$-integrin together with $\mathrm{C} 3 \mathrm{G}$ (Figure 7), suggesting that $\mathrm{C} 3 \mathrm{G}$ may play a role in regulation of $\beta$ PS-integrin localisation in muscles. 

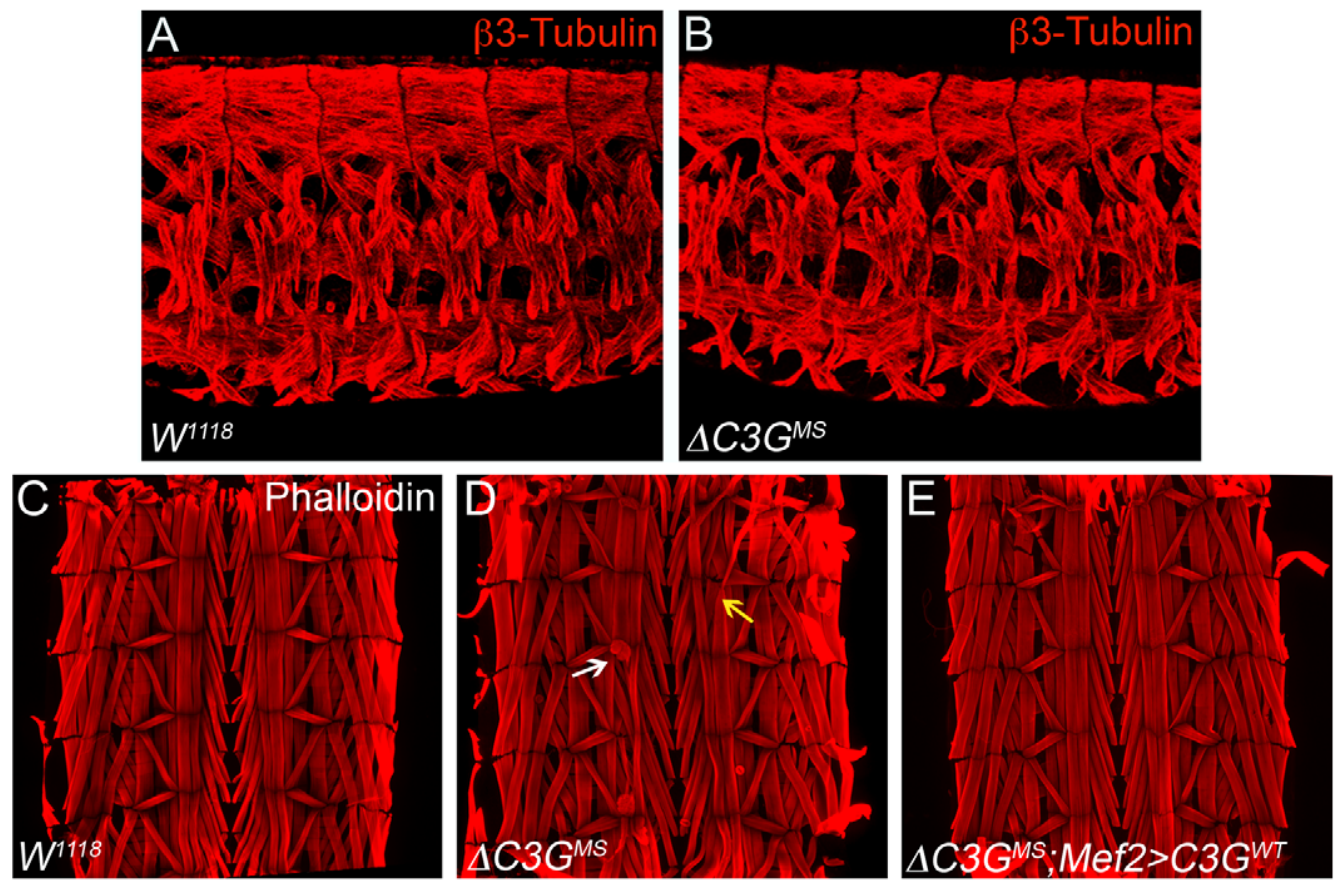

$\mathrm{F}$

\section{$\%$ Disruption of muscle integrity}
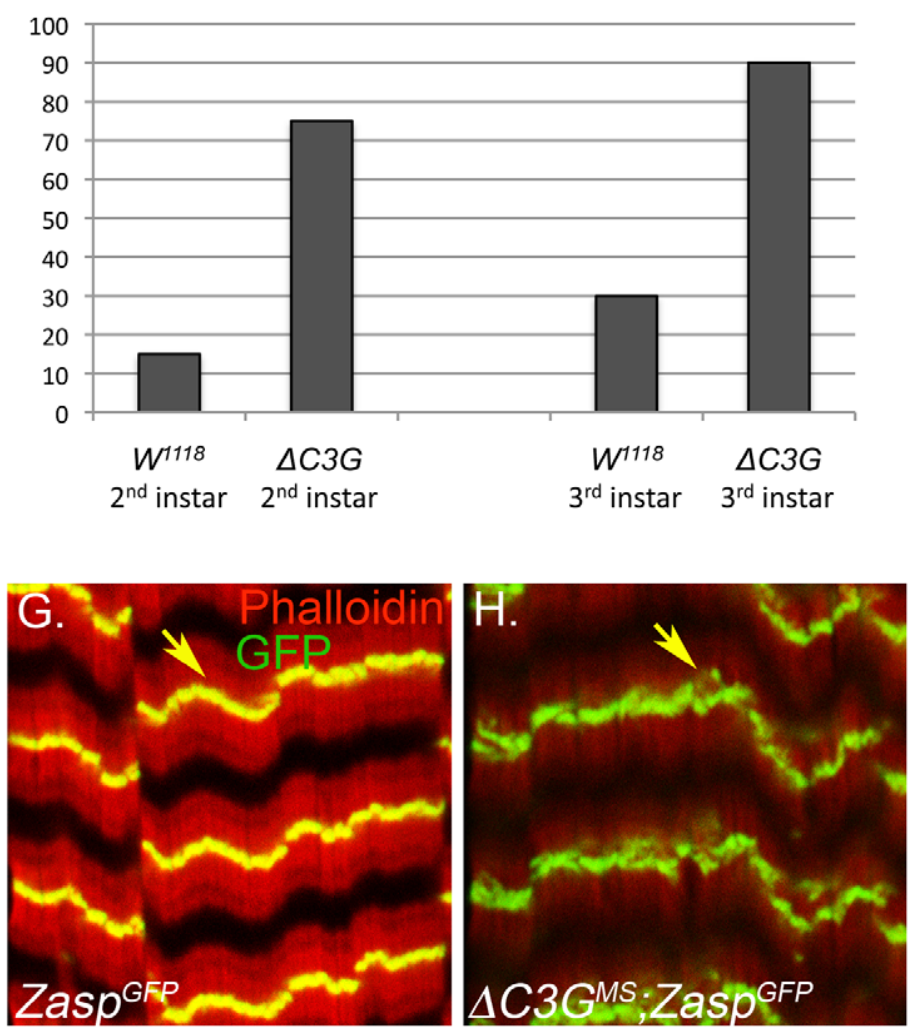

Figure 4. $\triangle C 3 G^{M S}$ mutant animals display unique somatic muscle phenotypes during larval development. Wild type and $A C 3 G^{M S}$ mutant embryos were stained with anti- $\beta 3$-tubulin antibodies (red) to visualize somatic muscles. (A, B) Similar to wild types, $\triangle C 3 G^{M S}$ mutants develop a normal somatic musculature at the end of embryogenesis. (C, D) Third instar $\Delta C 3 G^{M S}$ larvae exhibit morphological defects in the muscles (including muscle mistargeting and detachment as well as muscle "thinning", specifically in the ventral longitudinal muscles (D; arrows). (E) The somatic muscle defects observed in $\triangle C 3 G^{M S}$ mutant larvae can be rescued by GAL4/UAS-mediated ( $\triangle C 3 G ;$;ef2-GAL4 $>U A S-C 3 G^{W T}$ ) reintroduction of wild type C3G in muscles $(\mathbf{E})$. (F) Quantification of larval muscle phenotypes at the $2^{\text {nd }}$ and $3^{\text {rd }}$ instar larval stages, respectively. $(\mathbf{G}, \mathbf{H})$ Investigation of Z-band structures in wild type and $\triangle \mathcal{C} 3 G^{M S}$ mutants, utilizing a reporter for the Z-band specific protein Zasp, Zasp-GFP. Defects in Zasp-GFP distribution can be observed in $\triangle C 3 G^{M S}$ muscles $(\mathbf{H}$; arrows).

doi:10.1371/journal.pone.0009403.g004 

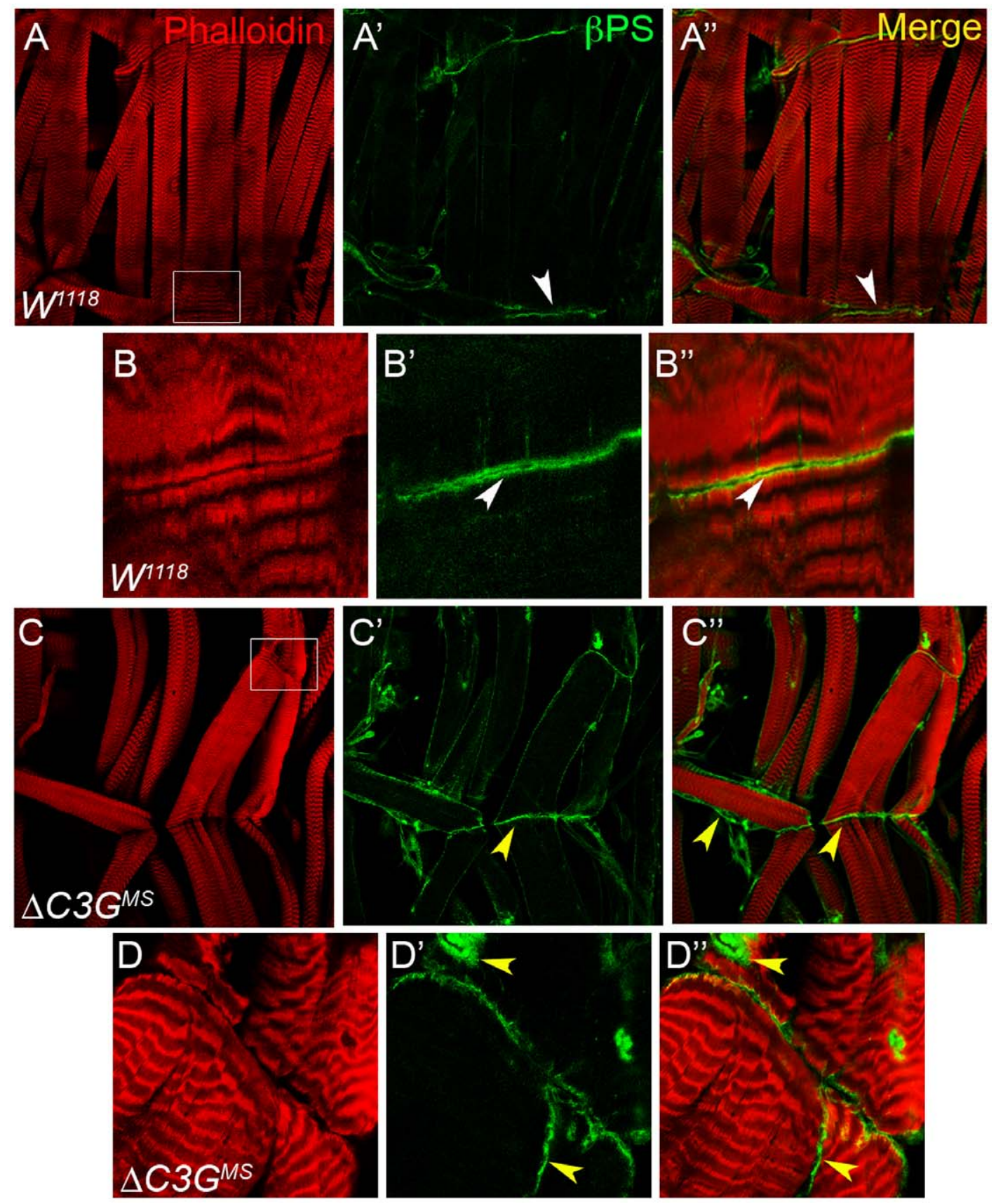

Figure 5. Localization of the $\beta P S$ integrin at the muscle attachment sites is affected in $\Delta C 3 G^{M S}$ mutant larvae. Third instar wild type control $(\mathbf{A}, \mathbf{B})$ and $\triangle C 3 G^{M S}$ mutant $(\mathbf{C}, \mathbf{D})$ larval muscles were dissected and stained with Phalloidin to visualize the muscles (red) and anti- $\beta P S$ integrin antibodies to depict muscle attachment sites (green). In contrast to wild type animals, the localization of $\beta$ PS integrins was not restricted to the attachment sites in $\triangle C 3 G^{M S}$ mutant muscles (yellow arrowheads). Instead of being concentrated at the attachment between two adjacent ventral longitudinal muscles $\left(\mathbf{C}^{\prime}, \mathbf{D}^{\prime}\right)$, $\beta$ PS was also visible around the ventral longitudinal muscle membrane (compare to $\mathbf{A}^{\prime}, \mathbf{C}^{\prime}$ ).

doi:10.1371/journal.pone.0009403.g005

\section{C3G Shows Specific GEF Activity towards Drosophila Rap1 but Not Rap2 In Vitro}

Drosophila contains two Rap proteins, both of which are putative targets for the GEF activities of C3G. To test if Drosophila C3G functions as a GEF for the Drosophila small GTPases Rapl and Rap2, we performed in vitro GDP release assays, using the RasGEF domain of $\mathrm{C} 3 \mathrm{G}$ together with Rap1 or Rap2. These assays establish that Drosophila C3G stimulates guanine nucleotide release from Drosophila Rap1, but not Rap2 (Figure 8A). Since Drosophila and human $\mathrm{C} 3 \mathrm{G}$ are closely related and share a high homology in their catalytic domains, we also examined whether human C3G acts as a GEF for Drosophila Rapl and Rap2. Similar to the Drosophila C3G, human C3G indeed stimulated guanine nucleotide release from Drosophila Rap1 but not Rap2 (Figure 8A). To demonstrate the general ability of our assay to identify GEF activity towards Rap2, RapGEF6 (PDZ-GEF2) was included as a positive control (Figure 8A). The reciprocal experiment was subsequently carried out to test whether Drosophila C3G shows any GEF activity towards human Rapl (hRap1B) and Rap2 (hRap2B) (Figure 8A). Drosophila G3G stimulates guanine nucleotide release 

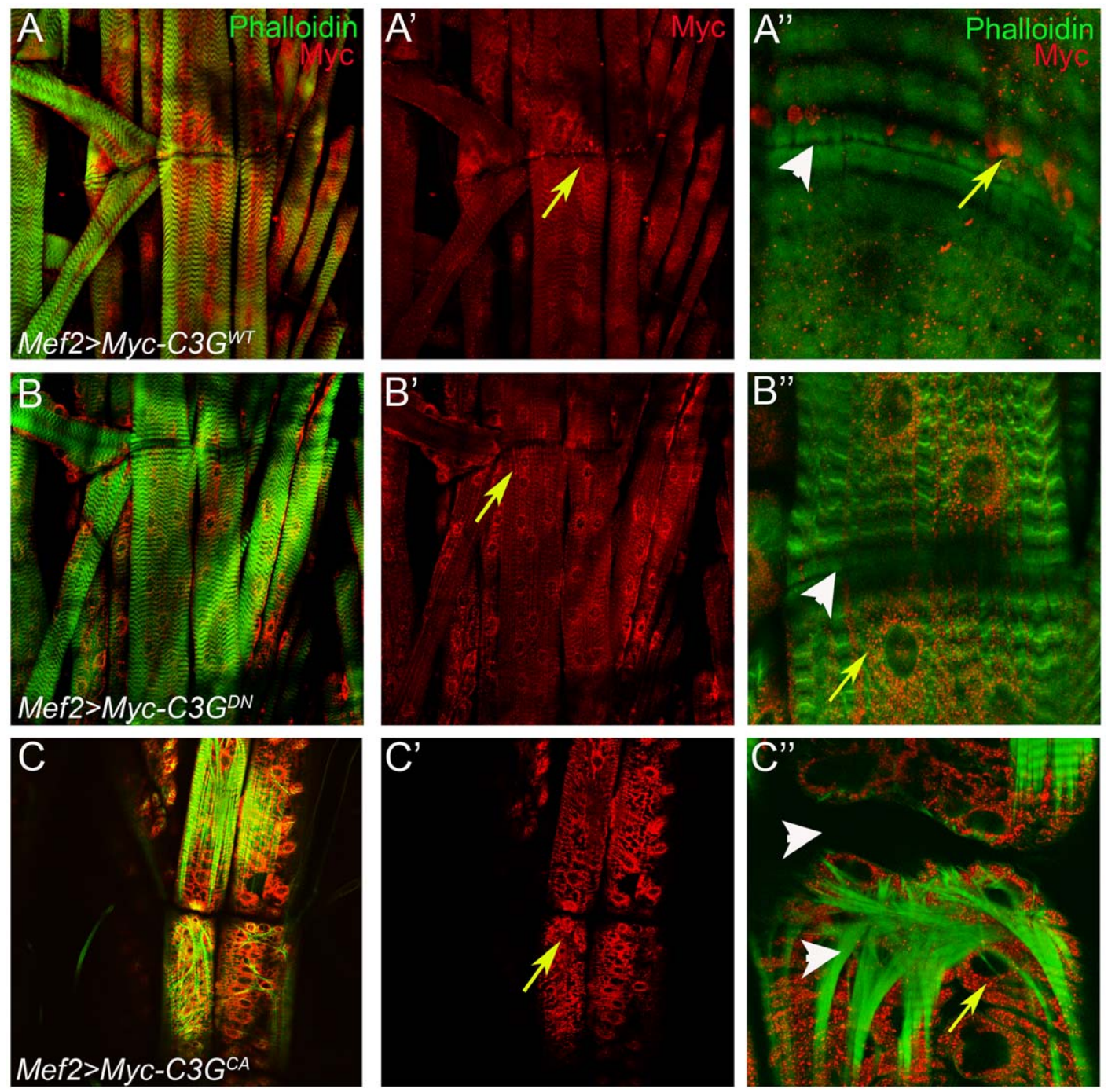

Figure 6. Overexpression of Drosophila C3G in larval muscles. Wild type $\left(\mathrm{C}^{\mathrm{W}} \mathrm{G}^{\mathrm{WT}}\right)$, dominant negative $\left(\mathrm{C} 3 \mathrm{G}^{\mathrm{DN}}\right)$ and activated $\left(\mathrm{C} 3 \mathrm{G}^{\mathrm{CA}}\right)$ myctagged variants of C3G were overexpressed in larval muscles using the muscle specific Mef2-Gal4 driver. Third instar larval muscles were dissected and stained with phalloidin (green) to visualize muscles and anti-myc antibodies to detect C3G (red). (A-A") Mef2-GAL4 induced C3GWT protein localizes around the muscle nuclei and additionally accumulates at one side of the attachment site between two ventral longitudinal muscles; arrows in $\left(\mathbf{A}^{\prime}, \mathbf{A}^{\prime \prime}\right)$. (B-B') Muscles overexpressing C3G ${ }^{\mathrm{DN}}$ mainly show a perinuclear localization of $C 3 \mathrm{G}^{\mathrm{DN}}$, resembling the pattern of C3G ${ }^{\mathrm{WT}}$; arrow in $\left(\mathbf{B}^{\prime}\right)$. (C-C') Muscles overexpressing the activated form of $C 3 \mathrm{G}\left(\mathrm{C} 3 \mathrm{G}^{\mathrm{CA}}\right)$; arrows $\left(\mathbf{C}^{\prime}, \mathbf{C}^{\prime \prime}\right)$ indicate localization of also $\mathrm{C}^{\prime} \mathrm{G}^{\mathrm{CA}}$ around the nuclei in the muscles. Muscle fibers appear disorganized. Arrowheads in $\left(\mathbf{C}^{\prime \prime}\right)$ indicate VL (Ventral Longitudinal) muscle detachment and the defects in actin filament structure.

doi:10.1371/journal.pone.0009403.g006

from human Rapl as well as Rap2, but activity towards Rap2 is less efficient than towards Rapl. Finally, we examined the GEF activity of Drosophila C3G towards H-Ras and could conclude that C3G does not stimulate efficient nucleotide exchange from H-Ras, in contrast with the established Ras-GEF Sos, which was employed as positive control (Figure 8B). Thus, at least in vitro, Drosophila C3G appears to act as a Rapl specific GEF.

\section{Discussion}

In this paper we report that loss of the guanine nucleotide exchange factor C3G in Drosophila causes semi-lethality, and that C3G null mutants $\left(\triangle C 3 G^{M S}\right)$ display defects in larval muscle architecture and decreased survival during both larval and adult phases. Both the muscle defects and lethality observed in $\Delta C 3 G^{M S}$ animals can be significantly rescued by the ectopic expression of wild type C3G protein in muscles with the Mef2-Gal4 driver, suggesting that the C3G GEF protein performs important functions in maintaining somatic muscle integrity. Our detailed analysis of the $\triangle C 3 G^{M S}$ mutants resulted in several important findings. Firstly, Drosophila C3G is crucial for the formation of normal larval body wall muscle structure, in particular the morphology and attachment of the ventral longitudinal muscles. During larval movement, it is the contraction of these longitudinal muscles, which alternately shorten the length of these segments uni- and bilaterally, that drives locomotion. The deformed morphology of these muscles may be the underlying explanation for the requirement of $\mathrm{C} 3 \mathrm{G}$ for larval fitness. One possibility is that the altered integrin localization in $\triangle C 3 G^{M S}$ mutants arises as a result of distorted muscle activity due to aberrant sarcomeric muscle structure. Indeed, in both Drosophila melanogaster and Caenorhabditis elegans integrins are required for proper sarcomere 

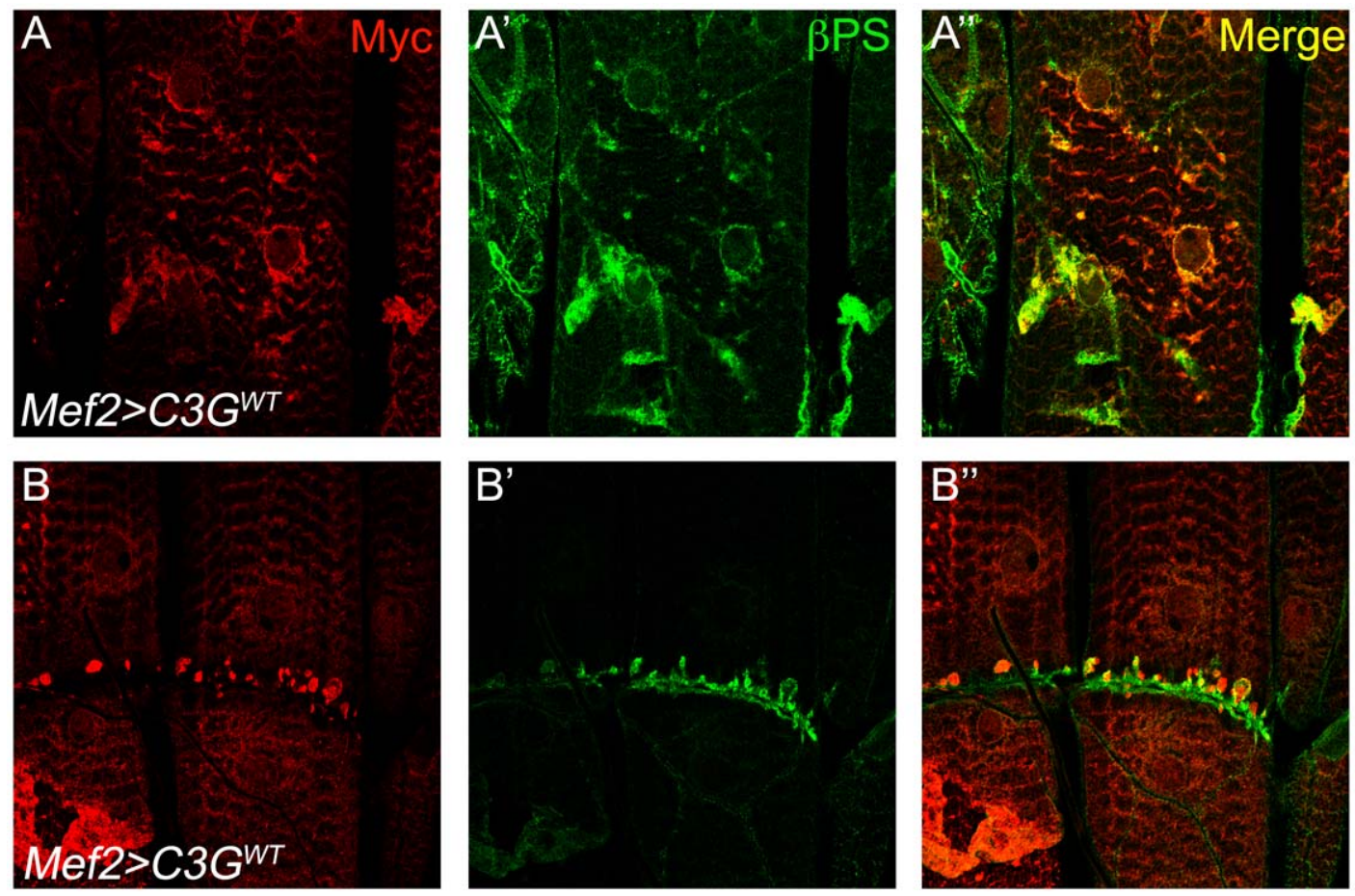

Figure 7. $\boldsymbol{\beta P S}$ integrins are recruited to sites of misexpressed C3G ${ }^{\mathbf{W T}}$ protein. (A-B) Mef2-GAL4 was used to drive expression of wild type $\left(C 3 G^{W T}\right)$ specifically in muscles. Body wall muscles from $3^{\text {rd }}$ instar larvae were dissected and stained with antibodies recognizing Myc-C3G ${ }^{\mathrm{WT}}$ (red) and $\beta P S$ (green), respectively. $\beta$ PS was observed to partially co-localize with $C 3 G^{W T}$, both in perinuclear regions (A- $\mathbf{A}^{\prime \prime}$ ) and in the accumulations of $C 3 \mathrm{G}^{\mathrm{WT}}$ protein at one side of muscle attachment sites $\left(\mathbf{B}-\mathbf{B}^{\prime \prime}\right)$.

doi:10.1371/journal.pone.0009403.g007

assembly and $\mathrm{Z}$ band formation [26,27,28,29]. In Drosophila absence of integrin function causes muscle detachment with no defects in the initial specification and fusion of muscle cells [26]. PS integrin deficiency has in cell culture systems been linked to loss of sarcomeric structures [27], thus suggesting a role for integrins in linking muscle attachment sites with the actin-myosin contractile apparatus [27,30,31,32]. In agreement, C3G deficient mice fibroblasts display cell adhesion defects and lack paxillin and $\beta 1$ integrin positive cell adhesions [8]. The panel of proteins involved in linking attachments and the sarcomeric muscle structures is constantly expanding. One such protein is the actin-binding protein $\alpha$-actinin. Drosophila $\alpha$-actinin mutant flies exhibit flight muscle paralysis [33], and indeed, upon close inspection using electron microscopy, mutant muscles show irregularities and dissolution of Z-bands, indicating a role for $\alpha$-actinin in anchoring and stabilizing the thin filaments of the muscles. Furthermore, a recent study in Drosophila has shown the PDZ-LIM domain containing protein Zasp to interact genetically with integrins, which is interesting given that zasp mutants do not form Z-bands and fail to recruit $\alpha$-actinin to the Z-band, resulting in muscle detachment at the onset of contractility [23]. Whereas Zasp localizes correctly at the Z-band in $\triangle C 3 G^{M S}$ mutants, the Z-band itself is irregular, raising the possibility that the integrin mislocalization observed in $\triangle C 3 G^{M S}$ mutant animals may partially be the result of defective anchorage of actin filaments to the $\mathrm{Z}$ bands. Other LIM domain adaptor proteins such as Paxillin and PINCH have also been proposed to strengthen integrin linkage to the actin cytoskeleton [34,35], but whether the localization of these molecules is affected in $\triangle C 3 G^{M S}$ mutants has not been analyzed.

In mammalian cells, endogenous C3G has been shown to interact with E-cadherin, a key component of cell-cell junctions, a binding that in the yeast-two-hybrid system was further mapped to the intracellular tail of E-cadherin [36]. The interaction between $\mathrm{C} 3 \mathrm{G}$ and E-cadherin is induced upon cell-cell junction disassembly and is temporally linked to the activation of Rapl, which has been proposed to occur at Rabl1-positive recycling endosomes [37]. Based on these findings, the internalization and disassembly of E-cadherin adhesive complexes may at a given point along the endocytic route expose the E-cadherin C3G binding site, enabling activation of Rapl, which appears to be a key determinant of E-cadherin recycling and formation of new cell-cell junction complexes [36,37]. Besides E-Cadherin, C3Gmediated activation of Rapl has been reported to occur also in response to other stimuli, including Nectin-mediated adhesion and mechanical stress. Nectins are immunoglobulin-like transmembrane proteins that can form homophilic or heterophilic complexes at the adherence junctions [38], supporting a role of Drosophila C3G at sites of cell-cell contact. The C3G-induced activation of Rapl in response to the mechanical stress emanating from cell stretching [39] has shown to be recognized by the p130Cas adaptor protein, which in turn recruits CRK, a constitutive binding partner of C3G. Taken together, this clearly demonstrates that C3G functions as an activator of Rapl and that this activation is involved in the regulation of cell adhesion. In agreement with this, Ishimaru and coworkers have reported that reduction of the gene dosage of Rapl or components of the Ras-MAPK pathway can reduce the phenotypes induced by ectopic C3G overexpression [13]. Muscle attachment sites are enriched with transmembrane proteins (primarily integrins), which are required for the initial establishment of attachments, as well as a multitude of proteins that function to stabilize the attachments by linking integrins to actin filaments, including Talin and $\alpha$-actinin $[23,33,34,35,40]$. Our data from the analysis 

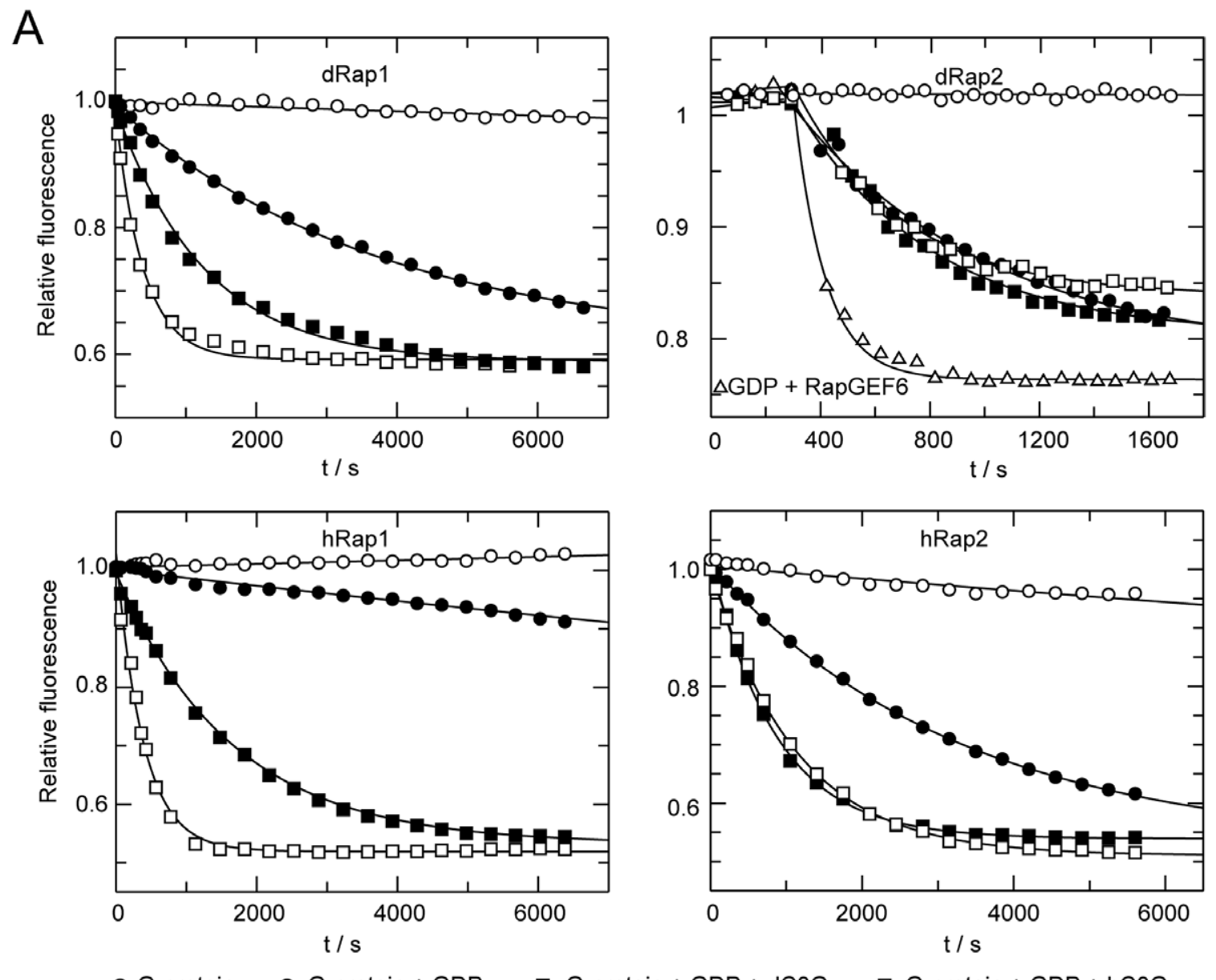

○ G-protein

- G-protein + GDP

G-protein + GDP + dC3G

G-protein + GDP + hC3G

B

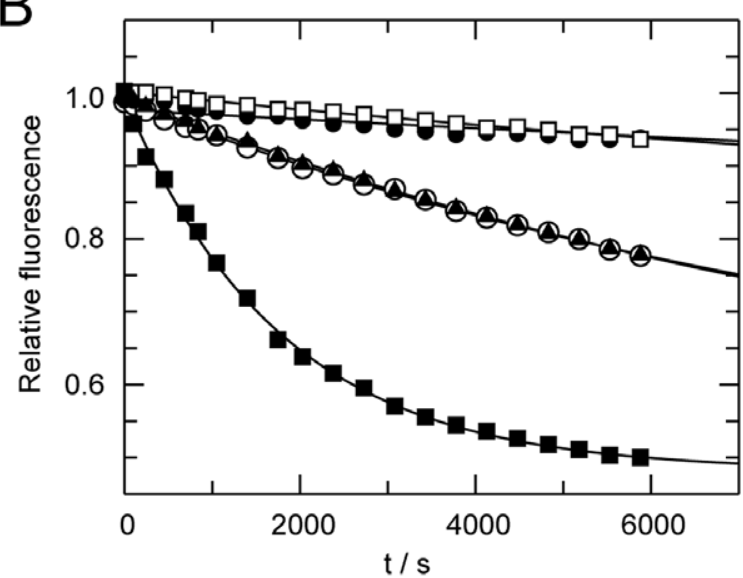

- HRas, $500 \mathrm{mM} \mathrm{NaCl}$

- HRas + GDP, $500 \mathrm{mM} \mathrm{NaCl}$

HRas + GDP, $100 \mathrm{mM} \mathrm{NaCl}$

- HRas + GDP + Sos, $100 \mathrm{mM} \mathrm{NaCl}$

^ HRas + GDP + dC3G, $500 \mathrm{mM} \mathrm{NaCl}$

Figure 8. Guanine nucleotide exchange rates of Drosophila and human Rap proteins are stimulated by C3G. (A, B) The guanine nucleotide exchange activity of Drosophila and human C3G were analysed in vitro. Briefly, GTPases were preloaded with the fluorescent GDP analogue mant-GDP. The fluorescence intensity of mant-GDP is approximately double that bound to the GTPase as free in solution. In the presence of an excess unlabelled normal GDP mant-GDP is exchanged for GDP, causing a decay of the fluorescence signal, allowing a direct measurement of the exchange rate. Given that the protein is stable, a constant fluorescence signal is expected in the absence of GDP. Addition of GDP monitors the intrinsic exchange rate of the GTPase, which should be enhanced in the presence of a GEF. (A) The exchange rates of Drosophila (upper panel) or human (lower panel) Rap1 and Rap2 were analysed in the presence or absence of either the Drosophila or human C3G RasGEF domain. Both Drosophila and human C3G stimulated nucleotide exchange from Drosophila Rap1, whereas Drosophila C3G did not exhibit activity towards Drosophila Rap2. RapGEF6 was employed as a positive control for GEF activity towards Drosophila Rap2. Drosophila C3G stimulated nucleotide release from both human Rap1 and less efficiently from human Rap2. (B) In contrast to human Sos, Drosophila C3G was unable to function as a GEF for H-Ras, confirming the function of Drosophila C3G as a Rap-GEF.

doi:10.1371/journal.pone.0009403.g008 
of $\Delta C 3 G^{M S}$ mutants, together with $\mathrm{C} 3 \mathrm{G}$ overexpression studies, suggests that $\mathrm{C} 3 \mathrm{G}$ acts as one such stabilizing protein required for preserving muscle integrity.

Our nucleotide exchange activity assays revealed that Drosophila C3G specifically exerts GEF activity towards Drosophila Rap1, but not markedly towards Rap2. Furthermore, both Drosophila and human C3G display an ability to stimulate nucleotide exchange of both human Rapl and, to a lesser extent, human Rap2B. Human C3G was previously shown not to act on human Rap2A [41], which implicates a conserved tendency of C3G to act more efficiently on Rap1 small GTPases than the Rap2 subfamily. This is the first in vitro evidence showing that Drosophila C3G selectively functions as a GEF for Rapl. The Drosophila genome contains one annotated Rap1 protein (roughened) and one Rap2 family member (rap2L) [42]. Besides C3G, the Drosophila PDZ-GEF (gef26) has also been reported as a GEF for Drosophila Rap1, and has been implicated in vivo in photoreceptor development as well as the regulation of cell migration and morphogenesis in wing disc epithelia, dorsal closure and hemocyte migration [43,44,45]. However, it has not been investigated whether Rapl has any role in the development of the larval musculature. Drosophila Rap2L has not been extensively studied in vivo and no mutants have been described at this point. Furthermore, it is not known which GEF proteins are responsible for the activation of Drosophila Rap2L, although our results indicate that it is unlikely to be activated directly by Drosophila C3G. In the GEF assays performed here we noted a high intrinsic exchange activity of Drosophila Rap2L, a feature which potentially could be explained by the slight sequence difference in the GTP-binding (NKXD) motif displayed by Drosophila Rap2L, as compared to the other Drosophila and human Rap proteins as well as most other Ras proteins.

The identification here of a function for $\mathrm{C} 3 \mathrm{G}$ in the somatic musculature adds another GEF to the list of GTPase modulators with described roles in muscle development. Previousely identified GEFs include Myoblast city (MBC) which functions as a GEF for Rac1, playing a critical role in the myoblast fusion process $[46,47]$. Additionally, the ARF6 GEF Loner also displays myoblast fusion defects [48]. To these we now add a role for the Rap GEF C3G in the regulation of the integrity of the larval musculature.

The punctuate and perinuclear localization of C3G upon overexpression is intriguing, although this pattern and its relevance for muscle integrity and attachment is unclear. In addition to their sarcomeric architecture, muscles contain tubular plasma membrane invaginations (transverse tubules or T-tubules) that are important for the connection between neuronal depolarization and muscle contraction. A number of proteins have been reported as critical for the biogenesis of these tubular structures. Two of these are Amphiphysin and Caveolin, which both have established functions in membrane dynamics $[49,50,51,52]$. It is conceivable that $\mathrm{C} 3 \mathrm{G}$ is also required for the normal biogenesis of T-tubules and if so, that the pattern of misexpressed activated C3G in muscles is the result of defective T-tubule formation. However, there is also a possibility that the punctuate pattern observed upon overexpression of wild type $\mathrm{C} 3 \mathrm{G}$ may be related to integrin trafficking events at the attachment sites. A number of recent studies in cell culture systems have provided insights into how integrin trafficking is regulated by the Rab family of small GTPases $[53,54]$, and it has additionally been shown that vesicular import of integrins is an essential event during cytokinesis. It is therefore imaginable that such a mechanism of integrin regulation also exists in Drosophila muscles.

Taken together, the data we have presented here provide the first in vivo evidence for a requirement of the guanine nucleotide exchange factor $\mathrm{C} 3 \mathrm{G}$ in the preservation of muscle integrity during larval development. Further studies are clearly needed to fully understand the function of $\mathrm{C} 3 \mathrm{G}$, explain the phenotypes observed in $\mathrm{C} 3 \mathrm{G}$ deficient animals, and uncover the molecular machinery that together with $\mathrm{C} 3 \mathrm{G}$ regulate muscle attachment, integrity, contractility and muscle maintenance.

\section{Supporting Information}

Figure S1 shifted is expressed in $\Delta C 3 G^{M S}$ mutants. RT-Reverse transcription PCR was performed on RNA extracts from wild type flies, flies carrying the starting transposable elements (RBe03301 and XPd00064) and $\Delta C 3 G^{M S}$ mutants. shf (shifted) is expressed in $\triangle C 3 G^{M S}$ mutants.

Found at: doi:10.1371/journal.pone.0009403.s001 (0.96 MB TIF)

Figure S2 $\triangle C 3 G^{M S}$ mutant embryos undergo normal fusion and migration of somatic and visceral muscles. To visualize the somatic muscle attachment sites, wild type and $\Delta C 3 G^{M S}$ mutant embryos were stained with $\beta$ PS integrin (red) and $\beta 3$-tubulin (green) antibodies. $\beta \mathrm{PS}$ integrin localization appears to be normal in $\Delta C 3 G^{M S}$ mutant embryos (A, B). (C, D) Wild type and $\Delta C 3 G^{M S}$ mutant embryos were stained with anti-Alk antibodies (green) to visualize the visceral muscles and anti-Connectin antibodies (red) as a cytoskeletal marker in the visceral mesoderm. The founder cells and fusion competent myoblasts fuse normally in $\Delta C 3 G^{M S}$ mutant embryos (D).

Found at: doi:10.1371/journal.pone.0009403.s002 (4.02 MB TIF)

Figure S3 $\Delta C 3 G^{M S}$ mutant larvae display muscle abnormalities already at the $2^{\text {nd }}$ instar stage of development. (A, B) Wild type and $\Delta C 3 G^{M S}$ mutant larvae were dissected at the $2^{\text {nd }}$ instar stage and stained with Phalloidin to visualize the body wall musculature. Whereas wild type animals (A) at this stage display regular and robust longitudinal muscle fibers, the $\Delta C 3 G^{M S}$ mutants (B) are characterized by thin longitudinal muscle fibers that tend to be both mistargeted and in some cases detached (right panel).

Found at: doi:10.1371/journal.pone.0009403.s003 (2.22 MB TIF)

Figure S4 $\Delta C 3 G^{M S}$ mutant muscles do not display increased apoptosis. Third instar wild type and $\Delta C 3 G^{M S}$ mutant larval muscles were dissected and subjected to a TUNEL assay. DNA fragmentation, characteristic for apoptosis, was analyzed by fluorescent labeling (Green). Samples treated with DNAse I were used as positive controls (A'). As negative control samples were treated with the TUNEL reaction mixture, but without addition of the terminal transferase. No apoptosis was observed in $\triangle C 3 G^{M S}$ mutant larva, similar to wild type muscles (D'-C').

Found at: doi:10.1371/journal.pone.0009403.s004 (7.43 MB TIF)

Figure S5 Embryonic muscle development is affected by C3G ${ }^{\mathrm{CA}}$, but not $\mathrm{C} 3 \mathrm{G}^{\mathrm{WT}}$ or $\mathrm{C} 3 \mathrm{G}^{\mathrm{DN}}$ misexpression. (A-D) Mef2-GAL4 was used to drive expression of wild type (C3G ${ }^{\mathrm{WT}}$ ), dominant negative $\left(\mathrm{C} 3 \mathrm{G}^{\mathrm{DN}}\right.$ ) or activated $\left(\mathrm{C} 3 \mathrm{G}^{\mathrm{CA}}\right.$ ) specifically in muscles. Late stages embryos were collected and stained with anti- $\beta 3$-tubulin (green) and anti- $\beta$ PS (red) antibodies. The integrity of the embryonic somatic musculature and the targeting of integrins to muscle attachment sites was not affected by misexpression of either C3G WT $(B)$ or C3G ${ }^{\text {DN }}(\mathrm{C})$. However, misexpression of $\mathrm{C} 3 \mathrm{G}^{\mathrm{CA}}$ resulted in muscle defects of variable magnitudes (D).

Found at: doi:10.1371/journal.pone.0009403.s005 (4.03 MB TIF)

\section{Acknowledgments}

The authors would like to thank members of the RHP and BH laboratory for helpful discussions during the course of this work and specifically thank 
Therese Eriksson and Gautam Kao for helpful comments and critical reading of the manuscript, Marije Rensen-De Leeuw for technical assistance and Camilla Sjögren for assistance with in situ analysis.

\section{References}

1. Pannekoek WJ, Kooistra MR, Zwartkruis FJ, Bos JL (2009) Cell-cell junction formation: the role of Rapl and Rapl guanine nucleotide exchange factors. Biochim Biophys Acta 1788: 790-796.

2. Bos JL, Rehmann H, Wittinghofer A (2007) GEFs and GAPs: critical elements in the control of small $\mathrm{G}$ proteins. Cell 129: 865-877.

3. Knudsen BS, Feller SM, Hanafusa H (1994) Four proline-rich sequences of the guanine-nucleotide exchange factor $\mathrm{C} 3 \mathrm{G}$ bind with unique specificity to the first Src homology 3 domain of Crk. J Biol Chem. pp 32781-32787.

4. Tanaka S, Morishita T, Hashimoto Y, Hattori S, Nakamura S, et al. (1994) $\mathrm{C} 3 \mathrm{G}$, a guanine nucleotide-releasing protein expressed ubiquitously, binds to the Src homology 3 domains of CRK and GRB2/ASH proteins. Proc Natl Acad Sci U S A 91: 3443-3447.

5. Gotoh T, Hattori S, Nakamura S, Kitayama H, Noda M, et al. (1995) Identification of Rap1 as a target for the Crk SH3 domain-binding guanine nucleotide-releasing factor C3G. Mol Cell Biol 15: 6746-6753.

6. Gotoh T, Niino Y, Tokuda M, Hatase O, Nakamura S, et al. (1997) Activation of R-Ras by Ras-guanine nucleotide-releasing factor. J Biol Chem 272: 18602-18607.

7. Ohba Y, Ikuta K, Ogura A, Matsuda J, Mochizuki N, et al. (2001) Requirement for C3G-dependent Rapl activation for cell adhesion and embryogenesis. Embo J 20: 3333-3341.

8. Voss AK, Gruss P, Thomas T (2003) The guanine nucleotide exchange factor C3G is necessary for the formation of focal adhesions and vascular maturation. Development 130: 355-367.

9. Voss AK, Krebs DL, Thomas T (2006) C3G regulates the size of the cerebral cortex neural precursor population. Embo J 25: 3652-3663.

10. Radha V, Rajanna A, Mitra A, Rangaraj N, Swarup G (2007) C3G is required for c-Abl-induced filopodia and its overexpression promotes filopodia formation. Exp Cell Res 313: 2476-2492.

11. Okino K, Nagai H, Nakayama H, Doi D, Yoneyama K, et al. (2006) Inactivation of Crk SH3 domain-binding guanine nucleotide-releasing factor (C3G) in cervical squamous cell carcinoma. Int J Gynecol Cancer 16: 763-771.

12. Dupuy AG, L'Hoste S, Cherfils J, Camonis J, Gaudriault G, et al. (2005) Novel Rapl dominant-negative mutants interfere selectively with $\mathrm{C} 3 \mathrm{G}$ and Epac. Oncogene 24: 4509-4520.

13. Ishimaru S, Williams R, Clark E, Hanafusa H, Gaul U (1999) Activation of the Drosophila C3G leads to cell fate changes and overproliferation during development, mediated by the RAS-MAPK pathway and RAP1. Embo J 18: $145-155$.

14. Thibault ST, Singer MA, Miyazaki WY, Milash B, Dompe NA, et al. (2004) A complementary transposon tool kit for Drosophila melanogaster using $\mathrm{P}$ and piggyBac. Nat Genet 36: 283-287.

15. Kopczynski CC, Davis GW, Goodman CS (1996) A neural tetraspanin, encoded by late bloomer, that facilitates synapse formation. Science 271: 1867-1870.

16. Patel NH (1994) Imaging neuronal subsets and other cell types in whole-mount Drosophila embryos and larvae using antibody probes. Methods Cell Biol 44: 445-487.

17. Loren CE, Englund C, Grabbe C, Hallberg B, Hunter T, et al. (2003) A crucial role for the Anaplastic lymphoma kinase receptor tyrosine kinase in gut development in Drosophila melanogaster. EMBO Rep 4: 781-786.

18. Englund C, Loren CE, Grabbe C, Varshney GK, Deleuil F, et al. (2003) Jeb signals through the Alk receptor tyrosine kinase to drive visceral muscle fusion. Nature 425: 512-516.

19. Leiss D, Hinz U, Gasch A, Mertz R, Renkawitz-Pohl R (1988) Beta 3 tubulin expression characterizes the differentiating mesodermal germ layer during Drosophila embryogenesis. Development 104: 525-531.

20. Campos-Ortega JA, Hartenstein V (1997) The Embryonic Development of Drosophila melanogaster. Berlin, Germany: Springer.

21. Rehmann H (2006) Characterization of the activation of the Rap-specific exchange factor Epac by cyclic nucleotides. Methods Enzymol 407: 159-173.

22. Lee HH, Norris A, Weiss JB, Frasch M (2003) Jelly belly protein activates the receptor tyrosine kinase Alk to specify visceral muscle pioneers. Nature 425: $507-512$.

23. Jani K, Schock F (2007) Zasp is required for the assembly of functional integrin adhesion sites. J Cell Biol 179: 1583-1597.

24. Hoffman EP, Brown RH Jr, Kunkel LM (1987) Dystrophin: the protein product of the Duchenne muscular dystrophy locus. Cell 51: 919-928.

25. Blake DJ, Weir A, Newey SE, Davies KE (2002) Function and genetics of dystrophin and dystrophin-related proteins in muscle. Physiol Rev 82: 291-329.

26. Brown NH (1993) Integrins hold Drosophila together. Bioessays 15: 383-390.

27. Volk T, Fessler LI, Fessler JH (1990) A role for integrin in the formation of sarcomeric cytoarchitecture. Cell 63: 525-536.

28. Schwander M, Leu M, Stumm M, Dorchies OM, Ruegg UT, et al. (2003) Betal integrins regulate myoblast fusion and sarcomere assembly. Dev Cell 4: 673-685.

\section{Author Contributions}

Conceived and designed the experiments: MS HB RP. Performed the experiments: MS CG MP GV FH HR. Analyzed the data: MS CG HB HR RP. Wrote the paper: MS CG RP.

29. Bloor JW, Brown NH (1998) Genetic analysis of the Drosophila alphaPS2 integrin subunit reveals discrete adhesive, morphogenetic and sarcomeric functions. Genetics 148: 1127-1142.

30. Ervasti JM (2003) Costameres: the Achilles' heel of Herculean muscle. J Biol Chem 278: 13591-13594.

31. Pardo JV, Siliciano JD, Craig SW (1983) A vinculin-containing cortical lattice in skeletal muscle: transverse lattice elements ("costameres") mark sites of attachment between myofibrils and sarcolemma. Proc Natl Acad Sci U S A 80: 1008-1012.

32. Reedy MC, Beall C (1993) Ultrastructure of developing flight muscle in Drosophila. I. Assembly of myofibrils. Dev Biol 160: 443-465.

33. Roulier EM, Fyrberg C, Fyrberg E (1992) Perturbations of Drosophila alphaactinin cause muscle paralysis, weakness, and atrophy but do not confer obvious nonmuscle phenotypes. J Cell Biol 116: 911-922.

34. Yagi R, Ishimaru S, Yano H, Gaul U, Hanafusa H, et al. (2001) A novel muscle LIM-only protein is generated from the paxillin gene locus in Drosophila. EMBO Rep 2: 814-820.

35. Clark KA, McGrail M, Beckerle MC (2003) Analysis of PINCH function in Drosophila demonstrates its requirement in integrin-dependent cellular processes. Development 130: 2611-2621.

36. Hogan C, Serpente N, Cogram P, Hosking CR, Bialucha CU, et al. (2004) Rapl regulates the formation of E-cadherin-based cell-cell contacts. Mol Cell Biol 24: 6690-6700.

37. Balzac F, Avolio M, Degani S, Kaverina I, Torti M, et al. (2005) E-cadherin endocytosis regulates the activity of Rapl: a traffic light GTPase at the crossroads between cadherin and integrin function. J Cell Sci 118: 4765-4783.

38. Fukuyama T, Ogita H, Kawakatsu T, Fukuhara T, Yamada T, et al. (2005) Involvement of the c-Src-Crk-C3G-Rapl signaling in the nectin-induced activation of Cdc42 and formation of adherens junctions. J Biol Chem 280: 815-825.

39. Tamada M, Sheetz MP, Sawada Y (2004) Activation of a signaling cascade by cytoskeleton stretch. Dev Cell 7: 709-718.

40. Brown NH, Gregory SL, Rickoll WL, Fessler LI, Prout M, et al. (2002) Talin is essential for integrin function in Drosophila. Dev Cell 3: 569-579.

41. van den Berghe N, Cool RH, Horn G, Wittinghofer A (1997) Biochemical characterization of C3G: an exchange factor that discriminates between Rapl and Rap2 and is not inhibited by Rap1A(S17N). Oncogene 15: 845-850.

42. Adams MD, Celniker SE, Holt RA, Evans CA, Gocayne JD, et al. (2000) The genome sequence of Drosophila melanogaster. Science 287: 2185-2195.

43. Lee JH, Cho KS, Lee J, Kim D, Lee SB, et al. (2002) Drosophila PDZ-GEF, a guanine nucleotide exchange factor for Rapl GTPase, reveals a novel upstream regulatory mechanism in the mitogen-activated protein kinase signaling pathway. Mol Cell Biol 22: 7658-7666.

44. Boettner B, Van Aelst L (2007) The Rap GTPase activator Drosophila PDZGEF regulates cell shape in epithelial migration and morphogenesis. Mol Cell Biol 27: 7966-7980.

45. Huelsmann S, Hepper C, Marchese D, Knoll C, Reuter R (2006) The PDZGEF dizzy regulates cell shape of migrating macrophages via Rap1 and integrins in the Drosophila embryo. Development 133: 2915-2924.

46. Erickson MR, Galletta BJ, Abmayr SM (1997) Drosophila myoblast city encodes a conserved protein that is essential for myoblast fusion, dorsal closure, and cytoskeletal organization. J Cell Biol 138: 589-603.

47. Rushton E, Drysdale R, Abmayr SM, Michelson AM, Bate M (1995) Mutations in a novel gene, myoblast city, provide evidence in support of the founder cell hypothesis for Drosophila muscle development. Development 121: 1979-1988.

48. Chen EH, Pryce BA, Tzeng JA, Gonzalez GA, Olson EN (2003) Control of myoblast fusion by a guanine nucleotide exchange factor, loner, and its effector ARF6. Cell 114: 751-762.

49. Lee E, Marcucci M, Daniell L, Pypaert M, Weisz OA, et al. (2002) Amphiphysin 2 (Bin 1) and T-tubule biogenesis in muscle. Science 297: 1193-1196.

50. Parton RG, Way M, Zorzi N, Stang E (1997) Caveolin-3 associates with developing T-tubules during muscle differentiation. J Cell Biol 136: 137-154.

51. McNally EM, de Sa Moreira E, Duggan DJ, Bonnemann CG, Lisanti MP, et al. (1998) Caveolin-3 in muscular dystrophy. Hum Mol Genet 7: 871-877.

52. Galbiati F, Engelman JA, Volonte D, Zhang XL, Minetti C, et al. (2001) Caveolin-3 null mice show a loss of caveolae, changes in the microdomain distribution of the dystrophin-glycoprotein complex, and t-tubule abnormalities. J Biol Chem 276: 21425-21433.

53. Pellinen T, Tuomi S, Arjonen A, Wolf M, Edgren H, et al. (2008) Integrin trafficking regulated by Rab21 is necessary for cytokinesis. Dev Cell 15: 371-385.

54. Caswell PT, Chan M, Lindsay AJ, McCaffrey MW, Boettiger D, et al. (2008) Rab-coupling protein coordinates recycling of alpha5betal integrin and EGFR1 to promote cell migration in 3D microenvironments. J Cell Biol 183: 143-155. 